\title{
TOPOLOGICAL PROPERTIES OF THE EFFICIENT POINT SET
}

\author{
BEZALEL PELEG
}

\begin{abstract}
Let $Y$ be a closed and convex subset of a Euclidean space. We prove that the set of efficient points of $Y, M(Y)$, is contractible. Furthermore, if $M(Y)$ is closed (compact) then it is a retract of a convex closed (compact) set. Our proof relies on the Arrow-Barankin-Blackwell Theorem. A new proof is supplied for that theorem.
\end{abstract}

1. Introduction. The study of efficient points of convex sets is expounded by many writers (see, e.g., [3], [2, pp. 306-310], [6, §12.3]). In particular, topological properties of the efficient point set are investigated in [3, pp. 73-78]. This paper is a further contribution on this topic: In $\S 4$ we prove that the set of efficient points $M(Y)$ of a closed and convex subset $Y$ of a Euclidean space is contractible. Furthermore, if $M(Y)$ is closed (compact) then it is a retract of a convex closed (compact) set.

Our proofs make use of the Arrow-Barankin-Blackwell Theorem [1, Theorem 1]. This theorem is generalized in infinite-dimensional spaces in [9], [7], [8], [5], and [4]. In $\S 3$ we offer a new proof of the ArrowBarankin-Blackwell Theorem. Our proof is advantageous over the original one in two respects: It is a "constructive" proof, unlike that of Arrow, Barankin, and Blackwell. Furthermore, it is easier to generalize to infinitedimensional spaces (see [7]).

I am indebted to M. Perles and M. Yaari for many helpful discussions.

2. Preliminaries. Let $E^{n}$ be the $n$-dimensional Euclidean space. If $x, y \in E^{n}$, then we write $x \geqq y$ if $x_{i} \geqq y_{i}$ for $i=1, \cdots, n . x>y$ if $x \geqq y$ and $x \neq y . x \gg y$ if $x_{i}>y_{i}$ for $i=1, \cdots, n$. We denote by $E_{+}^{n}$ the nonnegative cone of $E^{n}$, i.e., $E_{+}^{n}=\left\{x \mid x \in E^{n}\right.$ and $\left.x \geqq 0\right\}$. The scalar product of two members $x$ and $y$ of $E^{n}$ is denoted by $x \cdot y=\sum_{i=1}^{n} x_{i} y_{i}$. The norm of a member $x$ of $E^{n}$ is denoted by $\|x\|=(x \cdot x)^{1 / 2}, u^{(i)}, i=1, \cdots, n$, will denote the $i$ th unit vector of $E^{n}$.

3. An alternative proof of the Arrow-Barankin-Blackwell Theorem. Let $Y$ be subset of $E^{n}$. A point $e \in Y$ is an efficient point of $Y$ if there exists no

Received by the editors December 9, 1971 and, in revised form, January 31, 1972. AMS 1970 subject classifications. Primary 90A99; Secondary 90D12, 54F05. 
$y \in Y$ such that $y>e . r \in Y$ is a regular efficient point of $Y$ if there exists a vector $p \in E^{n}, p \gg 0$, such that $p \cdot r \geqq p \cdot y$ for all $y \in Y$. Clearly, a regular efficient point of $Y$ is an efficient point of $Y$. Let

$$
M(Y)=\{e \mid e \text { is an efficient point of } Y\} .
$$

Theorem 3.1 (ARrow, Barankin, AND BlackWell [1]). Let $Y$ be $a$ closed and convex subset of $E^{n}$. The regular efficient points of $Y$ are dense in $M(Y)$.

Proof. Let $e \in M(Y)$ and let $Y^{*}=\{y \mid y \in Y$ and $\|y-e\| \leqq 1\}$. If $r^{*}$ is a regular efficient point of $Y^{*}$ and $\left\|r^{*}-e\right\|<1$ then $r^{*}$ is a regular efficient point of $Y$. To see this let $p \gg 0$ satisfy $p \cdot r^{*} \geqq p \cdot y^{*}$ for all $y^{*} \in Y^{*}$. Let $y \in Y$. For $t>0$ sufficiently small, $t y+(1-t) r^{*} \in Y^{*}$. Hence, $p \cdot r^{*} \geqq$ $t \cdot p \cdot y+(1-t) p \cdot r^{*}$. Thus, $p \cdot r^{*} \geqq p \cdot y$. Thus, it is sufficient to prove that $e$ is the limit of a sequence of regular efficient points of $Y^{*}$. But $Y^{*}$ is compact. Hence, we may assume that $Y$ is compact. We may assume further that $Y \subset E_{+}^{n}$. Let $C=\max \{\|y\| \mid y \in Y\}$. For each $k, k=1,2, \cdots$, let

and

$$
\begin{gathered}
Y^{(k)}=\left\{y \mid y \in Y \text { and } y_{i} \geqq e_{i}-1 / k, i=1, \cdots, n\right\}, \\
v_{k}(x)=\min \left(x_{i}-e_{i}+1 / k, 1 \leqq i \leqq n\right), \quad x \in E^{n}, \\
w_{k}(x)=\sum_{i=1}^{n} x_{i} / n(k+1) C, \quad x \in E^{n},
\end{gathered}
$$

$$
u_{k}(x)=v_{k}(x)+w_{k}(x) .
$$

Let $r^{(k)}$ be a point where $u_{k}$ attains its maximum in $Y^{(k)} . u_{k}$ is concave; hence, the set

$$
Z=\left\{z \mid z \in E^{n} \text { and } u_{k}(z)>u_{k}\left(r^{(k)}\right)\right\}
$$

is convex. $Z \cap Y^{(k)}=\varnothing$. Hence, there exists a $p \in E^{n}$ such that

$$
p \cdot z \geqq p \cdot y \quad \text { for all } z \in Z \text { and } y \in Y^{(k)} \text {. }
$$

By (3.3), (3.4), and (3.5), $u_{k}$ is increasing, i.e., $x>y$ implies that $u_{k}(x)>$ $u_{k}(y)$. Hence, it follows from (3.6) and (3.7) that $p \gg 0$. Furthermore,

$$
p \cdot r^{(k)} \geqq p \cdot y \quad \text { for all } y \in Y^{(k)} \text {. }
$$

Since $1 / k \leqq u_{k}(e) \leqq u_{k}\left(r^{(k)}\right)$, it follows from (3.4) that

$$
r_{i}^{(k)}>e_{i}-1 / k, \quad i=1, \cdots, n .
$$


It follows from (3.8) and (3.9) that

$$
p \cdot r^{(k)} \geqq p \cdot y \quad \text { for all } y \in Y .
$$

Thus, $r^{(k)}$ is a regular efficient point of $Y$. Since $r^{(k)} \in Y^{(k)}, k=1,2, \cdots$, and $e$ is efficient, $e=\lim _{k \rightarrow \infty} r^{(k)}$.

4. A proof that the set of efficient points is contractible. Let $Y$ be a closed and convex subset of $E^{n}$ and let $M(Y) \neq \varnothing$ (see (3.1)).

LEMmA 4.1. There exist a vector $p \gg 0$ and a real number $v$ such that $p \cdot y \leqq v$ for all $y \in Y$.

Proof. By Theorem 3.1 there exists a regular efficient point of $Y$.

COROLlaRY 4.2. For each $x \in E^{n}$ the set $\{y \mid y \in Y$ and $y \geqq x\}$ is compact.

Lemma 4.3. Let $Y^{*}=\{x \mid$ there exists $y \in Y$ such that $y \geqq x\}$. Then $Y^{*}$ is convex and closed and $M(Y)=M\left(Y^{*}\right)$.

Proof. It is clear that $Y^{*}$ is convex and that $M(Y)=M\left(Y^{*}\right)$. To see that $Y^{*}$ is closed let $x=\lim _{k \rightarrow \infty} x^{(k)}, x^{(k)} \in Y^{*}, k=1.2 . \cdots$. There exist $y^{(k)} \in Y, y^{(k)} \geqq x^{(k)}, k=1,2, \cdots$. By Corollary 4.2 the sequence $\left(y^{(k)}\right)$ is bounded. Hence, we may assume that there exists a vector $y$ such that $y=\lim _{k \rightarrow \infty} y^{(k)}$. Clearly, $y \in Y$ and $y \geqq x$.

By Lemma 4.3 we may assume henceforth that $Y=Y^{*}$.

COROLlaRY 4.4. There exist points $a, b \in Y$ such that $h \gg a$.

For $y \in Y$ we define

$$
G(y)=\{x \mid x \in Y \text { and } x \geqq y\} .
$$

$G(y)$ is convex and compact. Also, $G$ is an upper semicontinuous function of $y$.

Lemma 4.5. Let $y \in Y$. If there exists a $z \in Y$ such that $z \gg y$ then $G$ is lower semicontinuous at $y$.

Proof. Let $y=\lim _{k \rightarrow \infty} y^{(k)}$ and let $x \in G(y)$. Let $1>t>0$.

$$
x(t)=t z+(1-t) x \gg y .
$$

Hence, there exists a natural number $k(t)$ such that $x(t) \subseteq G\left(y^{(k)}\right)$ for $k \geqq$ $k(t)$. Since $\lim _{t \rightarrow 0} x(t)=x$, the lemma follows.

We recall that a topological space is contractible if its identity map is homotopic to a constant.

THEOREM 4.6. $M(Y)$ is contractible.

Proof. Let $p$ and $v$ be as in Lemma 4.1. Let $w=\min _{1} p_{i}$. For $y \in Y$ let $f(y)=y+(v-p \cdot y) \sum_{i=1}^{n} u^{(i)} / w$. $f$ is a continuous function of $y$. 
Furthermore,

$$
f(y) \geqq x \quad \text { for all } x \in G(y) \quad(\text { see }(4.1)) .
$$

For $y \in Y$ let $g(y) \in G(y)$ be the point defined by $\|g(y)-f(y)\| \leqq\|x-f(y)\|$ for all $x \in G(y) . g(y)$ is well defined. By (4.2), $g(y) \in M(Y)$. Now let $a \in Y$ be a point for which there exists a $b \in Y$ such that $b \gg a$ (see Corollary 4.4). For $e \in M(Y)$ and $0 \leqq t \leqq 1$ let $h(e, t)=g((1-t) e+t a)$. $h(e, 0)=e$ and $h(e, 1)=g(a)$ for all $e \in M(Y)$. Furthermore, $h$ is a continuous function of both $e$ and $t$. For let $t=\lim _{k \rightarrow \infty} t^{(k)}$ and $e=\lim _{k \rightarrow \infty} e^{(k)}$. If $t=0$ then

$$
\begin{gathered}
\lim _{k \rightarrow \infty}\left(1-t^{(k)}\right) e^{(k)}+t^{(k)} a=e . \\
h\left(e^{(k)}, t^{(k)}\right) \geqq\left(1-t^{(k)}\right) e^{(k)}+t^{(k)} a .
\end{gathered}
$$

By Corollary 4.2 the sequence $\left(h\left(e^{(k)}, t^{(k)}\right)\right)$ is bounded. Hence, it follows from (4.3) and from $e \in M(Y)$ that $\lim _{k \rightarrow \infty} h\left(e^{(k)}, t^{(k)}\right)=e$. If $t>0$ then

$$
(1-t) e+(b)\rangle(1-t) e+t a=\lim _{k \rightarrow \infty}\left(1-t^{(k)}\right) e^{(k)}+t^{(k)} a .
$$

Hence, by Lemma 4.5, $G$ is lower semicontinuous at $(1-t) e+t a$. Therefore, $g$ is continuous at $(1-t) e+t a=y$. For assume, on the contrary, that

Then

$$
y=\lim _{k \rightarrow \infty} y^{(k)} \text { and } \lim _{k \rightarrow \infty} g\left(y^{(k)}\right)=z \neq g(y) .
$$

$$
\|g(y)-f(y)\|<\|z-f(y)\| .
$$

Furthermore, there exist $x^{(k)} \in G\left(y^{(k)}\right), k=1,2, \cdots$, such that

$$
\lim _{k \rightarrow \infty} x^{(k)}=g(y) \text {. }
$$

It follows from (4.4)-(4.6) that there exists a $k$ such that

$$
\text { : } x^{(k)}-f\left(y^{(k)}\right)\|<\| g\left(y^{(k)}\right)-f\left(y^{(k)}\right) \|,
$$

which is impossible. The continuity of $h$ at $(e, t)$ follows now from the continuity of $g$ at $(1-t) e+t a$.

We recall that a subset $A$ of a topological space $T$ is a retract of $T$ if there exists a continuous function $r: T \rightarrow A$ such that $r(a)=a$ for all $a \in A$.

THEOREM 4.7. If $M(Y)$ is closed then it is a retract of a closed and convex set. Let

Proof. For $y \leqslant Y$ let $d(y, M(Y))$ be the distance between $y$ and $M(Y)$.

$$
t(y)=d(y, M(Y)) /(1+d(y, M(Y))) .
$$


Then $t(y)$ is a continuous function of $y$ and $y \in M(Y)$ if and only if $t(y)=0$. Using the notation of the proof of Theorem 4.6 we define, for $y \in Y$,

$$
h(y)=g((1-t(y)) y+t(y) a) .
$$

Then $h(y) \in M(Y)$ and $h(e)=e$ for $e \in M(Y)$. Furthermore, it follows from the definition of $t(y)$ and Lemma 4.5 that $h$ is continuous. Hence, $h$ is a retraction of $Y$ on $M(Y)$.

THEOREM 4.8. If $M(Y)$ is compact then it is a retract of a compact and convex set.

Proof. "Choose $a \in E^{n}$ for which there exists $e \in M(Y)$ such that $e \gg a$. Let $Y_{1}$ be the convex hull of $M(Y) \cup\{a\}$. Then $Y_{1}$ is convex and compact and $M\left(Y_{1}\right)=M(Y)$. Let $q \in E^{n}$ satisfy $q \geqq x$ for all $x \in Y_{1}$. For $y \in Y_{1}$ let $g\left(y^{\prime}\right) \in G(y)=\left\{x \mid x \geqq y\right.$ and $\left.x \in Y_{1}\right\}$ be defined by

$$
\|g(y)-q\| \leqq\|x-q\| \quad \text { for all } x \in G(y) .
$$

Let further $t(y)$ be defined by (4.7). Define now, for $y \in Y_{1}$,

$$
h(y)=g((1-t(y)) y+t(y) a) .
$$

Then $h$ is a retraction of $Y_{1}$ on $M(Y)$.

REMARK 4.9. If $Y$ is polyhedral or strictly convex then $M(Y)$ is closed. However, $Y$ may be compact without $M(Y)$ being closed.

5. Concluding remarks. Let $Y$ be a closed and convex subset of a Euclidean space. Consider $Y$ as a technology given in the flow version (see $[6, \S 12])$. By Theorem 4.6, $M(Y)$ is contractible; hence, in particular, it is arcwise connected. Thus, it is possible to move from one efficient process to another via $M(Y)$ in a continuous manner. This result may be useful for economic planning.

If $Y$ is compact and polyhedral then, by Theorem 4.8, $M(Y)$ is a retract of a convex compact set. Therefore, every continuous function $f: M(Y) \rightarrow$ $M(Y)$ has a fixed point. This last fact may prove useful in game theory and mathematical economics investigations.

\section{REFERENCES}

1. K. J. Arrow, E. W. Barankin and D. Blackwell, Admissible points of convex sets, Contributions to the Theory of Games, vol. 2, Ann. of Math. Studies, nu. 28, Princeton Univ. Press, Princeton, N.J., 1953, pp. 87-91. MR 14, 998.

2. D. Gale, The theory of linear economic models, McGraw-Hill, New York, 1960. MR 22 \#6599.

3. T. C. Koopmans, Analysis of production as an efficient combınation of activities, Activity Analysis of Production and Allocation, Wiley, New York; Chapman and Hall, London, 1951. MR 13, 670. 
4. M. Kurz and M. Majumdar, Efficiency prices in infinite dimensional spaces: A synthesis (to appear).

5. M. Majumdar, Some approximation theorems on efficiency prices for infinite programs, J. Economic Theory 2 (1970), 399-410.

6. H. Nikaido, Convex structures and economic theory, Academic Press, New York, 1968.

7. B. Peleg, Efficiency prices for optimal consumption plans, J. Math. Anal. Appl. 29 (1970), 83-90. MR 41 \#5034.

8. - Efficiency prices for optimal consumption plans. II, Israel J. Math. 9 (1971), 222-234.

9. R. Radner, $A$ note on maximal points of convex sets in $l_{\infty}$, Proc. Fifth Berkeley Sympos. Math. Statist. and Probability (Berkeley, Calif., 1965/66), vol. 1: Statistics, Univ. of California Press, Berkeley, Calif., 1967, pp. 351-354. MR 35 \#7111.

Institute of Mathematics, HebreW University of Jerusalem, Jerusalem, IsRael 